\title{
Acetylation mediated by the p300/CBP-associated factor determines cellular energy metabolic pathways in cancer
}

\author{
RAMKUMAR RAJENDRAN ${ }^{1}$, RICHA GARVA ${ }^{2}$, HASSAN ASHOUR $^{2}$, TRAVIS LEUNG $^{1}$, IAN STRATFORD $^{1}$, \\ MARIJA KRSTIC-DEMONACOS ${ }^{2}$ and CONSTANTINOS DEMONACOS ${ }^{1}$ \\ ${ }^{1}$ School of Pharmacy and Pharmaceutical Sciences, Stopford Building and ${ }^{2}$ Faculty of Life Sciences, \\ Michael Smith Building, University of Manchester, Manchester, M13 9PT, UK
}

Received December 27, 2012; Accepted February 18, 2013

DOI: 10.3892/ijo.2013.1907

\begin{abstract}
Normal cells produce energy either through OXPHOS in the presence of oxygen or glycolysis in its absence. Cancer cells produce energy preferably through glycolysis even in the presence of oxygen, thereby, acquiring survival and proliferative advantages. Oncogenes and tumour suppressors control these metabolic pathways by regulating the expression of their target genes involved in these processes. During hypoxia, HIF-1 favours high glycolytic flux by upregulating glycolytic enzymes. Conversely, p53 inhibits glycolysis and increases OXPHOS expression through TIGAR and SCO2 gene expression, respectively. We hypothesise that the p300/ CBP-associated factor (PCAF) as a common co-factor shared between p53 and HIF-1 plays an important role in the regulation of energy production by modulating SCO2 and TIGAR gene expression mediated by these two transcription factors. The possible involvement of HIF-1 in the regulation of SCO2 and TIGAR gene expression was investigated in cells with different p53 status in normoxia- and hypoxia-mimicking conditions. Putative hypoxia response elements (HREs) were identified in the regulatory region of $S C O 2$ and TIGAR gene promoters. Chromatin immunoprecipitation experiments suggested that HIF-1 was recruited to the putative HREs present in the $S C O 2$ and TIGAR promoters in a cell type-dependent manner. Transcriptional assays endorsed the notion that PCAF may be involved in the determination of the SCO2 and TIGAR cellular levels, thereby, regulating cellular energy metabolism, a view
\end{abstract}

Correspondence to: Dr Constantinos Demonacos, School of Pharmacy and Pharmaceutical Sciences, Stopford Building, University of Manchester, Oxford Road, Manchester, M13 9PT, UK

E-mail: cdemonacos@manchester.ac.uk

Dr Marija Krstic-Demonacos, Faculty of Life Sciences, Michael Smith Building, University of Manchester, Oxford Road, Manchester, M13 9PT, UK

E-mail: m.k.demonacos@manchester.ac.uk

Key words: cancer, metabolism, p300/CBP associated factor, p53, hypoxia inducible factor, acetylation, glycolysis, oxidative phosphorylation supported by assays measuring lactic acid production and oxygen consumption in cells ectopically expressing PCAF. The present study identified HIF-1 as a potential regulator of SCO2 and TIGAR gene expression. Furthermore, evidence to suggest that PCAF is involved in the regulation of cellular energy production pathways in hypoxia-mimicking conditions is presented. This effect of PCAF is exerted by orchestrating differential recruitment of HIF-1 $\alpha$ and p53 to the promoter of $T I G A R$ and/or SCO2 genes, thereby, tailoring physiological needs and environmental conditions to SCO2 and TIGAR gene expression.

\section{Introduction}

It is well established that unlike normal tissues cancer cells switch the pathway of energy production from oxidative phosphorylation (OXPHOS) to aerobic glycolysis under normoxic conditions (Warburg effect) even though the efficiency of ATP production through OXPHOS is much higher than that of glycolysis $(1,2)$. The high demand of rapid energy release in fast proliferating cancer cells is covered more efficiently by glycolysis, since this pathway can be regulated effectively without requiring the replenishment of suitable metabolites as is the case with the aerobic respiration which is a slower process. In addition, glycolysis confers the ability to cancer cells to evade apoptosis, resist immune responses and use the glycolytic intermediate products as substrates for anabolic reactions, explaining the preference of these cells to produce energy through glycolysis (3-5).

Cellular energy needs are covered by the coordination of the gene expression of a network of metabolic genes carried out by oncogene and tumour suppressor pathways coupling environmental conditions to cellular physiology. For example, in conditions of low oxygen concentration, which is a common event in the tumour microenvironment, HIF-1, the major orchestrator of transcription under these conditions, induces gene expression of glycolytic enzymes and reduces mitochondrial activity switching the energy production pathway from oxidative phosphorylation to glycolysis thus facilitating the cellular adaptation to hypoxia (6-8).

The p53 tumour suppressor on the other hand, displays diverse effects on energy metabolism by fine tuning the gene expression of proteins involved in both OXPHOS and 
glycolysis $(9,10)$. Recently synthesis of cytochrome $c$ oxidase 2 (SCO2) and TP53-induced glycolysis and apoptosis regulator (TIGAR), two genes involved in oxidative phosphorylation and glycolysis respectively, have been documented as p53 transcriptional targets $(11,12)$. SCO2 is involved in the assembly of the cytochrome $c$ oxidase complex (complex IV subunit 2) in the mitochondrial respiratory electron transport chain and the delivery of copper to this complex (13). Mutations in $\mathrm{SCO} 2$ gene severely impair cytochrome $c$ oxidase assembly accompanied by cellular copper deficiency which result in various mitochondrial diseases such as encephalomyopathy and hypertrophic cardiomyopathy (14). By inducing $S C O 2$ gene expression, p53 enhances mitochondrial respiration $(9,10)$ while in its absence glycolysis prevails (11). TIGAR inhibits glycolysis as it displays homology to the phosphatase domain of the 6-phosphofructo-2-kinase/fructose-2,6-bisphosphatase (PFK2/FBPase2) enzyme which restrains glycolysis by dephosphorylating fructose-2,6-bisphosphate (F2,6BP). The kinase domain of the PFK2/FBPase2 induces the phosphorylation of fructose 6-phosphate (F6P) thereby increasing the intracellular levels of the $\mathrm{F} 2,6 \mathrm{BP}$, whereas the phosphatase domain of this bi-functional enzyme dephosphorylates F2,6BP to F6P. F2,6BP is an allosteric activator of PFK-1 therefore its levels play a crucial role in the determination of the glycolytic rate, the blockade of the Warburg effect and the redirection of glycolysis to the pentose phosphate pathway (PPP) $(3,15,16)$. Induction of TIGAR gene expression is an alternative way by which p53 determines the cellular fate by depleting the intracellular NADPH hence mediating antioxidant defence $(3,12)$.

We have previously demonstrated the role of the p300/ $\mathrm{CBP}$ associated factor (PCAF) as common coregulator of the function of p53 and HIF- $1 \alpha$ in hypoxia indicating that the acetylation function of PCAF is crucial in determining cell cycle arrest and survival or apoptosis in hypoxic conditions in a manner depending on its intrinsic HAT activity (17). PCAF mediated acetylation and Sirt-1 dependent deacetylation of HIF-1 $\alpha$ have been more recently shown to modulate cellular responses in hypoxia (18). Taking into account the fact that both PCAF and p300 interact with Sirt-1 $(19,20)$ and Sirt-1 is a well-known cellular sensor of reduced glucose and $\mathrm{NAD}^{+}$ availability (21), we hypothesised that PCAF as common regulator of $\mathrm{p} 53$ and HIF- $1 \alpha$, might coordinate the pathways for cellular energy production regulating the physiologically distinct pathways of OXPHOS and glycolysis.

It is known that p53 and HIF-1 $\alpha$ play important roles in the determination of sensitivity/resistance of cancer cells to chemotherapy or radiotherapy (22). In this respect, p53 degradation, or HIF- $1 \alpha$ stabilisation and fine tuning of their transcription target selectivity contribute to the response to radiation damage and affect the therapeutic efficacy of agents that regulate these properties. It is therefore of crucial importance to study the factors that can distinguish between the beneficial and detrimental properties of both these transcription factors in cancer therapy in order to improve the efficacy of various therapeutic approaches. We present herein evidence that the HAT activity of PCAF beyond its role in the control of cell proliferation and survival/apoptosis in hypoxic conditions (17) is also implicated in the modulation of metabolic pathways, such as OXPHOS or glycolysis regulating energy and oxidative stress homeostasis in cancer cells. This function of
PCAF is in accord with the recently presented view suggesting the existence of common regulatory systems shared between cell cycle progression and metabolic pathways (23).

\section{Materials and methods}

Cell lines, cell culture and constructs. U2OS and MCF7 (p53 wt) and MDA-MB-231 (p53 mutant) and SaOS2 (p53--) cells were cultured in DMEM (Sigma-Aldrich, UK) supplemented with $10 \% \mathrm{v} / \mathrm{v}$ heat inactivated fetal calf serum (Gibco, UK) and $1 \%$ of penicillin and streptomycin $10,000 \mathrm{U} / \mathrm{ml}$ (Lonza, USA) at $37^{\circ} \mathrm{C}$ in a humidified atmosphere containing $5 \% \mathrm{CO}_{2}$. Wherever mentioned, cells were treated with $250 \mu \mathrm{M}$ desferrioxamine (DSFX) (Sigma-Aldrich) for $16 \mathrm{~h}$.

The PCDNA3-Flag-TIGAR expression construct was a generous gift from Professor K. Vousden (Beatson Institute, Glasgow, UK) the PCDNA3-SCO2 expression construct was provided by Professor P. Hwang (NIH, Bethesda, MD, USA) and the pCiFlag-PCAF(wt) and pCiFlag-PCAF $(\triangle H A T)$ were obtained from Dr I. Talianidis (Athens, Greece) (24). Human SCO2 and TIGAR luciferase reporters containing the consensus HREs and 553 binding sites were constructed by amplifying the -287 to -1712 fragment of the $\mathrm{SCO} 2$ and the +400 to -508 region of the TIGAR (counted from the translation initiation codon) (primer sequences are shown in Table I) and inserting them in the pGL3 promoter luciferase vector (Promega, USA). The calcium phosphate method (25) and the polyfect transfection system (Qiagen, UK) were used to transfect cells. Luciferase reporter assays were carried out as described previously (25).

Immunoblotting and antibodies. Cells were harvested in $240 \mathrm{mM}$ TNN buffer (50 mM Tris-HCl pH 7.4, $240 \mathrm{mM} \mathrm{NaCl}$, $5 \mathrm{mM}$ EDTA and $0.5 \%$ NP-40) and equal amounts of protein were loaded and resolved by SDS-PAGE and western blotting. After incubating with primary and secondary antibodies, the blots were developed with ECL substrate according to the manufacturer's instructions (Pierce, Thermo Scientific, USA). The following antibodies were used for western blotting: $\beta$-actin (Abcam, UK), SCO2 (ProSciInc., USA), TIGAR-IN1 (ProSci Inc.), HIF-1 $\alpha$ (H1a67; Calbiochem, EMD Chemicals, USA), p53 (DO1; Santa Cruz Biotechnology, USA), PCAF (E-8; Santa Cruz Biotechnology), anti-Flag (M2; SigmaAldrich) and PFKFB3 (Abgent, USA).

Quantitative RT-PCR. Quantitative RT-PCR analysis was carried out as described previously (17). Briefly, total RNA was extracted from cells using RNeasy plus mini kit (Qiagen, USA) following the manufacturer's instructions. The RNA was then reverse transcribed to cDNA and used for qPCR analysis using SYBR Green fluorescent probe. Analysis was performed using the Opticon Monitor (Bio-Rad Laboratories, USA) or Realplex (Eppendorf, UK) software. The primer sequences used in qPCR reaction are provided in Table II.

Chromatin immunoprecipitation. Chromatin immunoprecipitation analysis was performed in U2OS cells as described previously (17). Briefly, chromatin was cross-linked using $1 \%$ formaldehyde and the protein DNA complex was sonicated to produce $\sim 500$ bp DNA fragments which were then immuno- 
Table I. Luciferase primers.

\begin{tabular}{lll}
\hline Gene name & \multicolumn{1}{c}{ Forward primer } & \multicolumn{1}{c}{ Reverse primer } \\
\hline TIGAR & 5'-CTCGAGGGGTGGGTGGGTCTAAGTCT-3' & 5'-GAGCTCGGACGAGCAATTCTGCAAAC-3' \\
SCO2 & 5'-ACCGTGGAGCTGGTCC-3' & 5'-CAGCAAGGTGAACCTCT-3' \\
\hline
\end{tabular}

Table II. qPCR primers.

\begin{tabular}{llll}
\hline Gene & Orientation & Primer sequence & Product size (bp) \\
\hline TIGAR & Forward & 8' & \\
& Reverse & 5'-ATGGAATTTTGGAGAGAA-3' & 149 \\
SCO2 & Forward & 5'-CCATGGCCCTCAGCTCAC-3' & \\
& Reverse & 5'-TCACTCACTGCCCTGACATC-3' & 122 \\
Rpl19 & Forward & 5'-CGGTCAGACCCAACAGTCTT-3' & \\
& Reverse & 5'-ATGTATCACAGCCTGTACCTG-3' & 5'-TTCTTGGTCTCTTCCTCCTTG-3' \\
\hline
\end{tabular}

Table III. ChIP primers.

\begin{tabular}{lll}
\hline & \multicolumn{1}{c}{ Forward } & Reverse \\
\hline TIGAR & & \\
HRE 1 & 5'-GTGTTGGAATCTCGGCTCAC-3' & 5'-CAAGGCAGGTCAGGAGAATC-3' \\
HRE 2 & 5'-CCGTGTTAGCCAAAATGGTC-3' & 5'-CACATTTGGCCTTCTGAACA-3' \\
HRE 3 and E2F1 & 5'-CTATAGAAGGGTGCGTCCTT-3' & 5'-TGACTCCTTCCCATTACCTA-3' \\
HRE 4 & 5'-ACACGGTGAAACCCTGTCTC-3' & 5'-AGTGCAGTGGTGTGATCTCG-3' \\
HRE 5 & 5'-CACAGTCTGTTGGTCGCTG-3' & 5'-GATTCCTTCCCTCGATAGCC-3' \\
HRE 6 & 5'-AGGAATCCTACCGCGGACT-3' & 5'-CTACCTCCCCCACACCACT-3' \\
SCO2 & & \\
HRE 1 & 5'-TGGTGCTGCACGAGCTCGG-3' & \\
HRE 2 & 5'-CTCTGCAGGGACCCCCTGGC-3' & \\
HRE 3 & 5'-CAGGAGGCGCTCGTACTCT-3' & 5'-GCGGTCGGAGAGTACGAGCG-3' \\
HRE 4 & 5'-CATGCGCAGCTCCGGGGAC-3' & 5'-GACAGGCTCTCAGCGCGTGC-3' \\
HRE 5 and p53 & 5'-GCCAGAGAGTTACCCACCTCCTTTTAA-3' & 5'-ACGAGAGGAAGCGCCGACCT-3' \\
HRE6 & 5'-GTGTGGTTGCCCAGGTGTGGA-3' & 5'-CTGTCACCGCACCCTGCCC-3' \\
\hline
\end{tabular}

precipitated with HIF-1 $\alpha$-ChIP grade (AB2185; Abcam) or an irrelevant antibody (HRP conjugated anti-rabbit). The reverse cross-linked DNA fragments were then amplified in PCR reactions with specific primers (Table III) flanking different HREs within the TIGAR and SCO2 promoter and analyzed using Opticon Monitor software (Bio-Rad Laboratories) or $2 \%$ agarose gel electrophoresis.

Measurement of oxygen consumption. A Clark-type oxygen electrode system (Rank Brothers, Cambridge, UK) was used to measure the ability of the cells to uptake oxygen. The Clarke type electrode uses polarising voltage to create a current or flow of electrons between one silver and one platinum electrode. The output of the electrode is connected to a voltage adaptor and the output of this adaptor is connected to a Pico Log recorder (Pico Technology Ltd., UK). As the oxygen concentration in the incubation chamber changes, the current flowing between the two metal electrodes changes in proportion of the oxygen concentration in the incubation chamber. These changes in the current are converted to changes in the voltage which was recorded in the Pico Log recorder. Cells $\left(2 \times 10^{5}\right)$ were collected in 2-ml medium loaded into the incubation chamber maintained at $37^{\circ} \mathrm{C}$ and stirred continuously using a magnetic stirrer. The value of the voltage indicating the amount of oxygen available in the incubation chamber at each time-point in DSFX-treated cells was divided with the respective value 

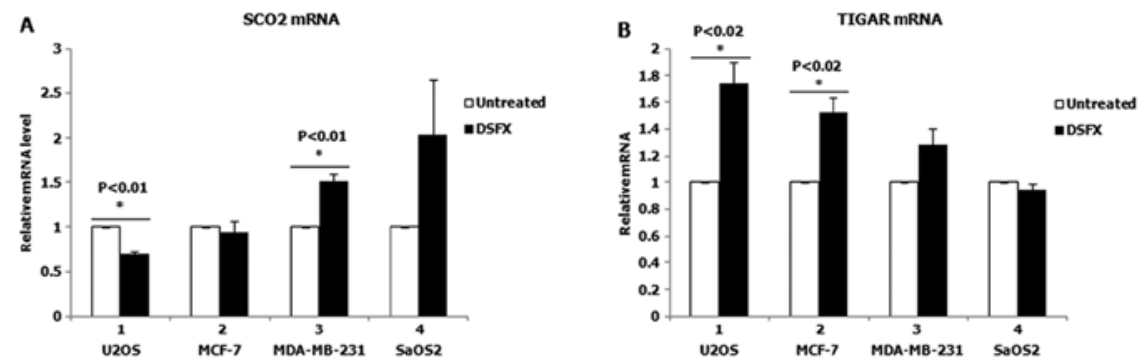

C

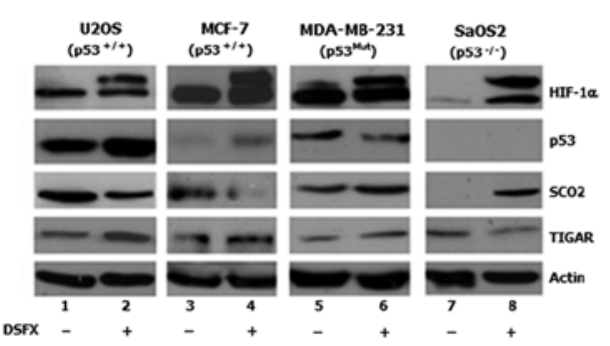

D

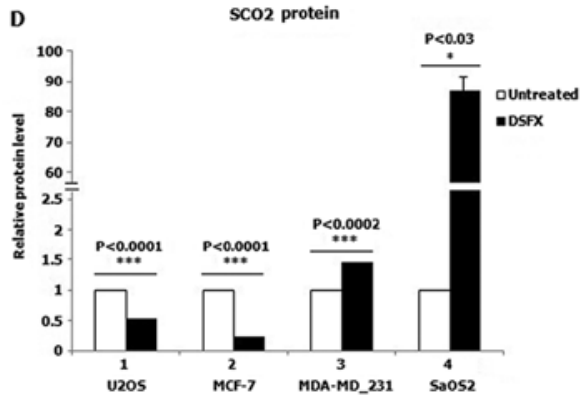

E

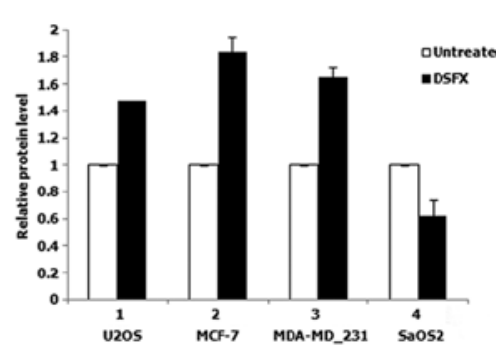

Figure 1. Regulation of SCO2 and TIGAR gene expression in hypoxia mimicking conditions. SCO2 (A) and TIGAR (B) mRNA and protein (C) levels were followed by qRT-PCR and western blotting, respectively, in non-treated or DSFX-treated U2OS, MCF-7, MDA-MB-231 and SaOS2 cells. Rp119 was used as an internal control in mRNA assays and actin as loading control in western blotting. The mRNA levels of SCO2 and TIGAR in non-treated cells was normalised to 1 (white bars). Densitometric analysis of SCO2 and TIGAR protein levels is shown in (D) and (E) respectively. Asterisks indicate statistical significance of the results.

obtained for the amount of oxygen available in the chamber in untreated cells. The inverted numbers attained from this calculation showing the oxygen consumption in treated and non-treated cells were plotted as a slope. Oxygen consumption was measured from 1 to $600 \mathrm{sec}$.

Measurement of lactic acid production. Cells were grown in 6-well plates, transiently transfected and treated as described in the text and figure legends. Lactate levels were quantified using Lactate Reagent kit (Trinity Biotech, Ireland) following the manufacturer's instructions. Lactate production rates were expressed as $\mathrm{nmol} / \mathrm{min}$ per milligram protein.

\section{Results}

SCO2 and TIGAR gene expression in hypoxia mimicking conditions. To investigate whether gene expression of the p53 transcription targets SCO2 and TIGAR was differentially affected in hypoxia mimicking conditions, we followed SCO2 and TIGAR mRNA and protein levels in p53wt (U2OS and MCF7), p53 mutated (MDA-MB-231), or p53-deficient (SaOS2) cells treated with the hypoxia mimicking agent DSFX. Significantly decreased SCO2 mRNA levels were observed in DSFX-treated U2OS cells (p53wt) compared to those in the non-treated cells (Fig. 1A, compare black bar 1 to white bar 1). In contrast, increased SCO2 mRNA levels were monitored in DSFX-treated MDA-MB-231 cells (mutated p53) and SaOS2 cells (p53 null) compared to those in non-treated cells (Fig. 1A, compare black bars 3 and 4 to white bars 3 and 4). Similarly lower SCO2 protein levels were detected in DSFX-treated versus non-treated U2OS and MCF-7 cells (Fig. 1C, compare lanes 2 to 1 and 4 to 3; Fig. 1D, compare black bars 1 and 2 to white bars 1 and 2) and higher in DSFX-treated as opposed to non-treated MDA-MB-231 and SaOS2 cells (Fig. 1C, compare lanes 6 to 5 and 8 to 7; Fig. 1D, compare black bars 3 and 4 to white bars 3 and 4).

In contrast to SCO2, increased TIGAR mRNA and protein levels were recorded in DSFX-treated U2OS and MCF-7 compared to non-treated cells (Fig. 1B, compare black bars 1 and 2 to white bars 1 and 2; Fig. 1C, compare lanes 2 to 1 and 4 to 3 ; Fig. 1E, compare black bars 1 and 2 to white bars 1 and 2). Although TIGAR mRNA levels did not change, downregulation of TIGAR protein was observed in DSFX-treated SaOS2 cells compared to non-treated cells (Fig. 1C, compare lanes 8 to 7 ; Fig. 1E, compare black bar 4 to white bar 4). Higher TIGAR mRNA and protein levels were documented in DSFXtreated MDA-MB-231 cells compared to those in non-treated cells (Fig. 1B, compare black bar 3 to white bar 3; Fig. 1C, compare lanes 6 to 5; Fig. 1E, compare black bar 3 to white bar 3). Taken together results presented in Fig. 1 are suggestive of a differential regulation of the SCO2 and TIGAR cellular levels in hypoxia mimicking conditions in a manner dependent on the presence of $\mathrm{p} 53$.

Putative HREs are present in the regulatory region of the $S C O 2$ and TIGAR promoters. To gain a better understanding of the transcriptional events regulating SCO2 and TIGAR gene expression in DSFX-treated cells and in particular to test whether HIF-1 was involved in these events, the upstream regulatory region of the $S C O 2$ and TIGAR promoters were submitted to bioinformatics analysis to investigate whether hypoxia responsive elements (HREs) were present in these regions. Putative HRE sites were identified within the regulatory region of both $S C O 2$ and TIGAR promoters (Fig. 2).

The presence of binding sites for several transcription factors in addition to the known p53 binding sites $(11,16)$ 
A

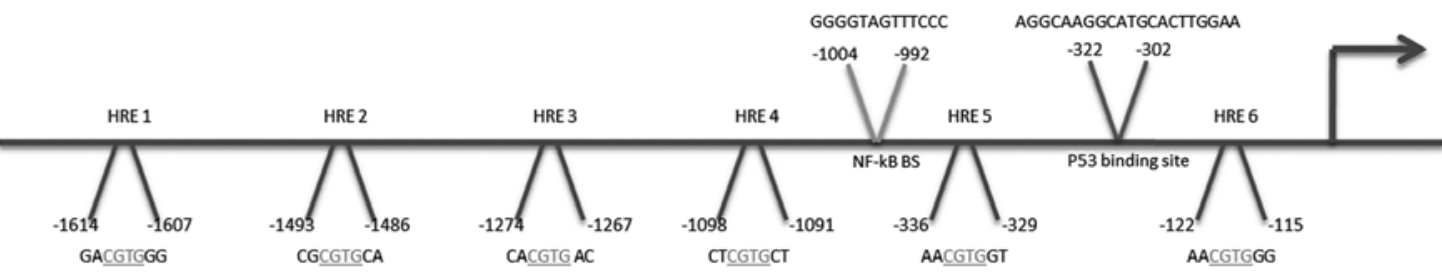

B

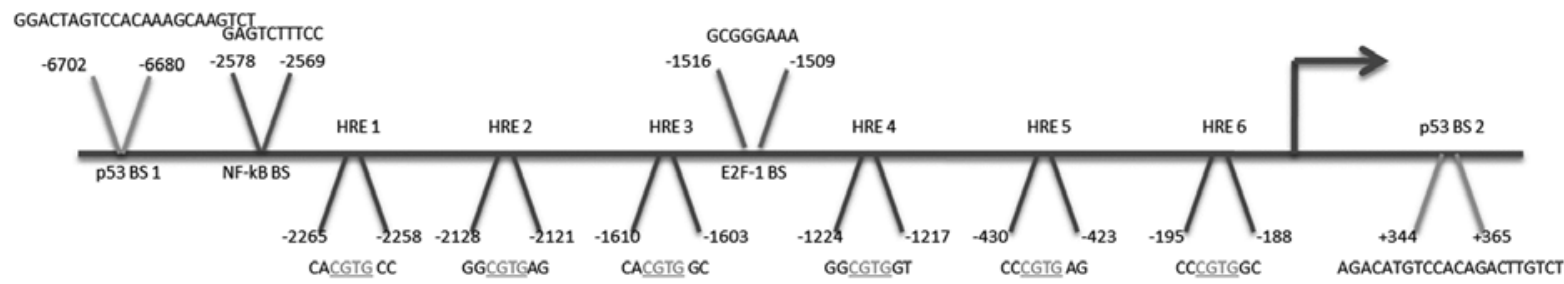

Figure 2. Schematic diagram indicating the HRE and p53 binding sites within the upstream regulatory region of the SCO2 (A) and TIGAR (B) promoters.
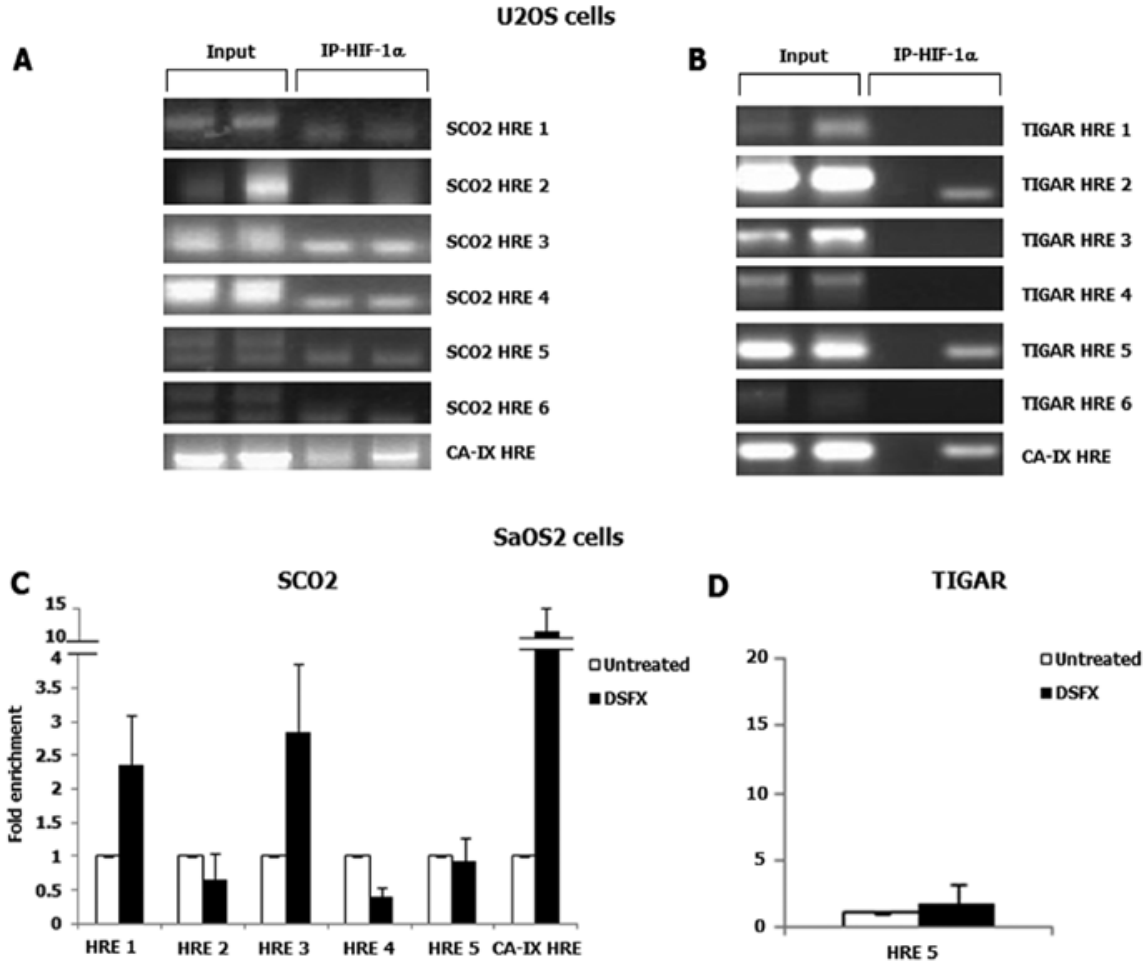

D

TIGAR

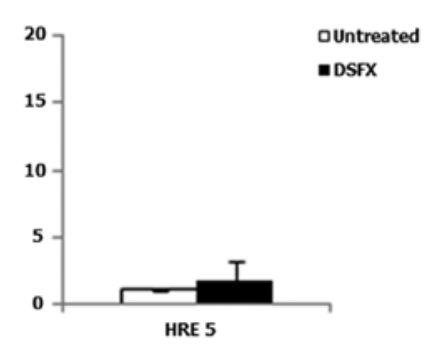

Figure 3. HIF-1 $\alpha$ is recruited to the promoters of SCO2 and TIGAR in a cell type-dependent manner. HIF-1 $\alpha$ bound to chromatin was immunoprecipitated from cellular extracts of non-treated or DSFX-treated U2OS (A and B) and SaOS2 (C and D) cells. The immunoprecipitated chromatin was amplified using specific primers flanking various regions containing the putative binding sites for HIF-1 in SCO2 (A and C) and TIGAR (B and D) promoter regions. Rabbit IgG was used as a negative control and 10\% input as loading and positive control. One representative out of three independent experiments is shown in (A) and (B). The average of two independent experiments performed in duplicates is shown in (C) and the average of three experiments performed in duplicates in (D).

within the upstream regulatory region of $S C O 2$ and TIGAR promoters implies that gene expression of these genes is under a complex control of multiple transcription factors. ChIP assays were carried out to assess the recruitment of HIF-1 to the putative binding sites identified in SCO2 and TIGAR promoters. HIF-1 complexes with chromatin were immunoprecipitated using HIF- $1 \alpha$ specific ChIP grade antibody. Rabbit IgG was used as a negative control and $10 \%$ input as loading control. The known HIF-1 transcription target gene
$C A-I X$ was used as a positive control (Fig. 3A-C). In U2OS cells HIF- $1 \alpha$ recruitment was not detected in any of the putative HREs identified within the SCO2 promoter (Fig. 3A), whereas only the HRE 5 within the TIGAR promoter was found to be occupied by HIF-1 $\alpha$ in DSFX-treated U2OS cells (Fig. 3B, compare lanes 3 with 4; the band appearing in the TIGAR HRE 2 panel is not specific). However, in $\mathrm{SaOS} 2$ cells, HIF-1 $\alpha$ was found to be recruited in the putative HRE 1 and HRE 3 sites within the SCO2 promoter (Fig. 3C). 


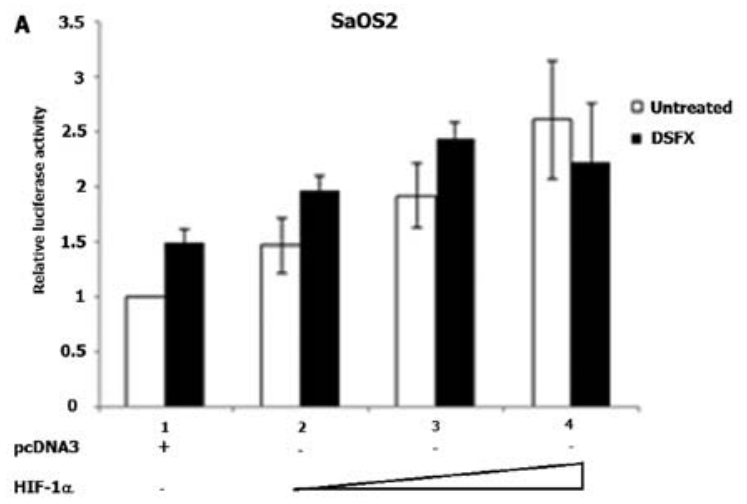

B
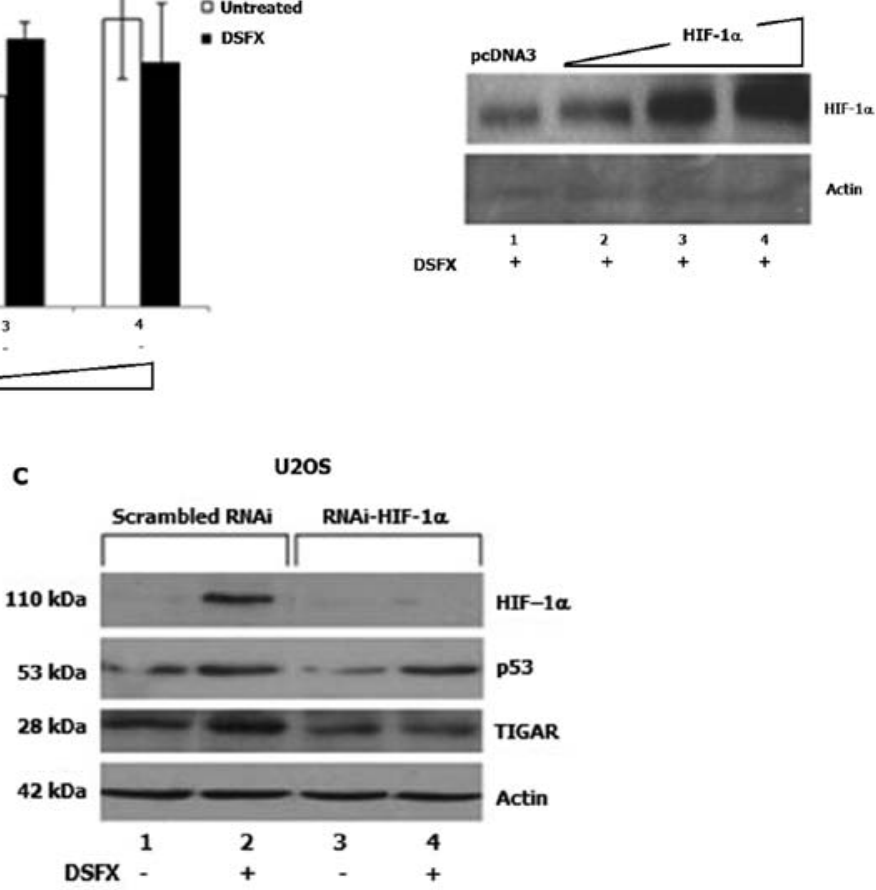

Figure 4. SCO2 and TIGAR are possible HIF-1 $\alpha$ transcription targets. (A) SCO2-HRE1-5-p53-Luc reporter assay in SaOS2 cells overexpressing increasing amounts of HIF-1 $\alpha$ were left untreated (white bars) or treated with DSFX (black bars) and subjected to luciferase reporter assays. (B) Cells transfected as described in (A) were submitted to western blotting and probed for HIF-1 $\alpha$. Actin was used as a loading control. (C) TIGAR protein levels in U2OS cells transfected with either scrambled RNAi (tracks 1 and 2) or RNAi against HIF-1 $\alpha$ (tracks 3 and 4). The cells were left untreated (lanes 1 and 3) or treated with DSFX (lanes 2 and 4). Actin was used as a loading control.

Furthermore, HIF-1 $\alpha$ was not detected bound to the HRE 5 of the TIGAR promoter in the Saos2 cells (Fig. 3D).

To further investigate the involvement of HIF-1 in the transcriptional regulation of $\mathrm{SCO} 2$ gene expression, the fragment of the SCO2 promoter containing the putative HREs 1-5 (Fig. 2A) was cloned in the pGL3-Luc reporter vector as described in Materials and methods. The constructed SCO2HRE1-5-p53bs-Luc was used to carry out luciferase reporter assays in DSFX-treated U2OS and SaOS2 cells ectopically expressing increasing amounts of HIF-1 $\alpha$. Results shown in Fig. 4A indicated that increasing amounts of HIF-1 $\alpha$ (Fig. 4B) induced SCO2-HRE1-5-p53bs-Luc expression in SaOS2 cells signifying the potential involvement of HIF-1 $\alpha$ in the regulation of SCO2 gene expression.

Possible involvement of HIF-1 $\alpha$ in the regulation of TIGAR gene expression was evidenced in U2OS cells transfected with RNAi against HIF-1 $\alpha$ and monitoring TIGAR protein levels (Fig. 4C). Increased TIGAR protein levels were observed in DSFX-treated U2OS cells transfected with scrambled RNAi compared to those detected in non-treated cells (Fig. 4C, compare lanes 2 to 1 ), whereas reduced TIGAR protein levels were recorded in U2OS cells transfected with RNAi-HIF (Fig. 4C, compare lanes 3 and 4 to lanes 1 and 2, respectively).

The HAT activity of PCAF is potentially involved in the determination of SCO2 and TIGAR cellular levels. We have previously reported that PCAF HAT activity plays important role in the determination of both p53 and HIF-1 $\alpha$ transcription target selectivity in hypoxia mimicking conditions thus coor- dinating cell cycle arrest or apoptosis under these conditions (17). It is also known that lysine acetylases (such as p300 and PCAF) and deacetylases (such as Sirt-1) act antagonistically to promote or suppress the transcriptional activity of many transcription factors including p53 and HIF-1, thereby regulating important cellular functions $(19,20)$.

Since $S C O 2$ and TIGAR were identified as possible common transcription targets of p53 $(11,16)$ and HIF-1 $\alpha$ (Figs. 1-4) we were intrigued to examine whether the HAT activity of PCAF played coordinative role in selectively targeting p53 or HIF-1 to the SCO2 and TIGAR promoter in response to diverse environmental stimuli. For this purpose, $\mathrm{SCO} 2$ protein levels were followed in non-treated and DSFX-treated U2OS and SaOS2 cells transfected with either, Flag-PCAFwt or Flag-PCAFAHAT expression vectors (Fig. 5). Reduced SCO2 protein levels were detected in DSFX-treated U2OS cells overexpressing Flag-PCAFwt compared to non-treated cells (Fig. 5A, compare lanes 2 to 1) while increased $\mathrm{SCO} 2$ protein levels were observed in DSFX-treated U2OS overexpressing Flag-PCAFAHAT compared to the non-treated cells (Fig. 5A, compare lanes 4 to 3). In Flag-PCAF $\Delta H A T$ overexpressing $\mathrm{SaOS} 2$ cells, reduced $\mathrm{SCO} 2$ protein levels were identified in DSFX-treated cells compared to the non-treated cells (Fig. 5D, compare lanes 4 to 3). No change in SCO2 protein levels was observed in Flag-PCAFwt overexpressing SaOS2 cells treated or not treated with DSFX (Fig. 5D, compare lanes 2 to 1).

Flag-PCAFwt overexpression in DSFX-treated U2OS cells downregulated luciferase expression driven by the SCO2-HRE1-5-p53bs-Luc reporter (Fig. 5B, compare bars 


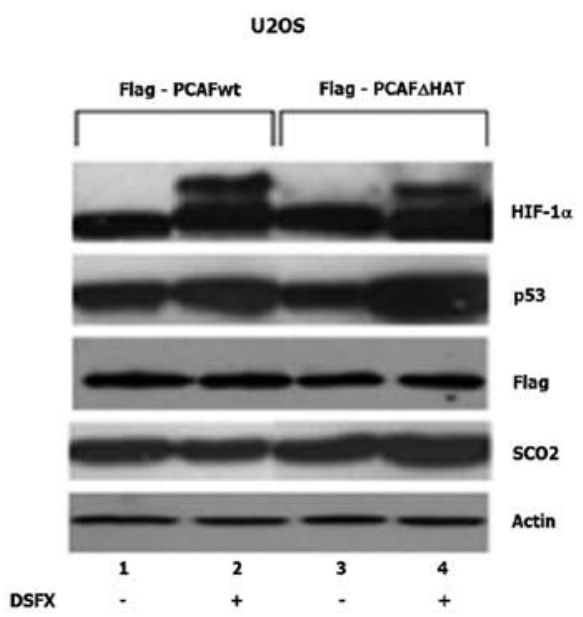

B

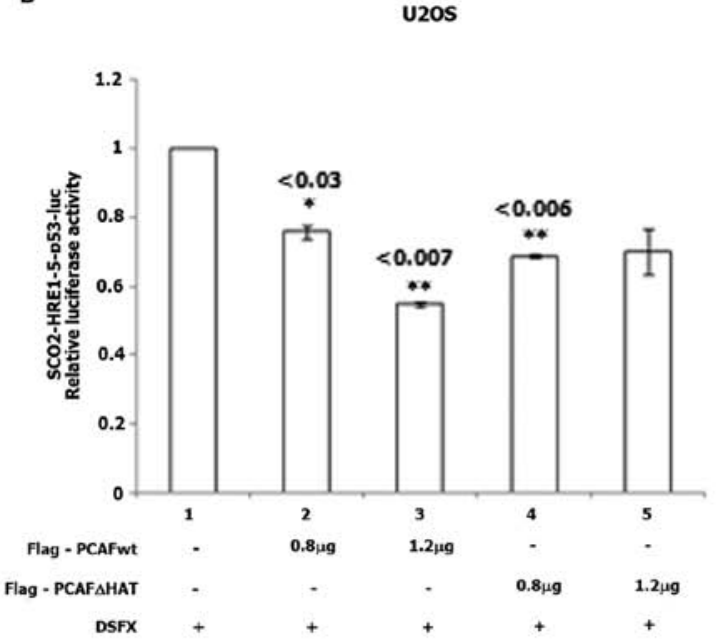

c

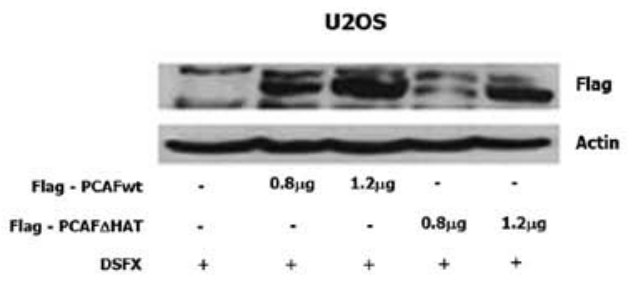

D

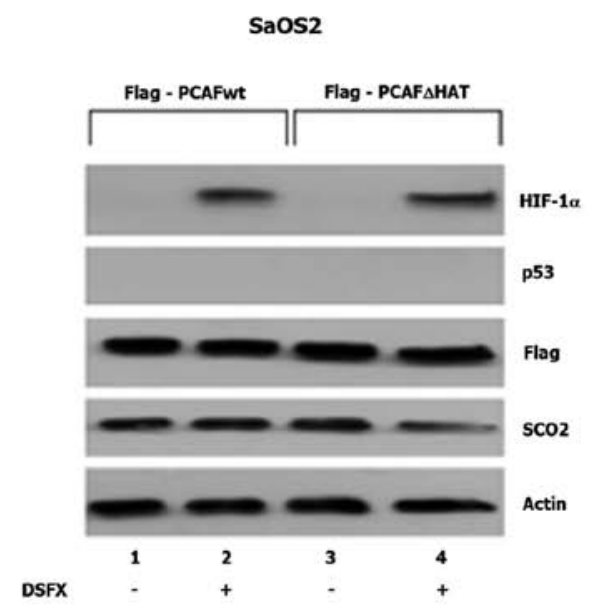

E

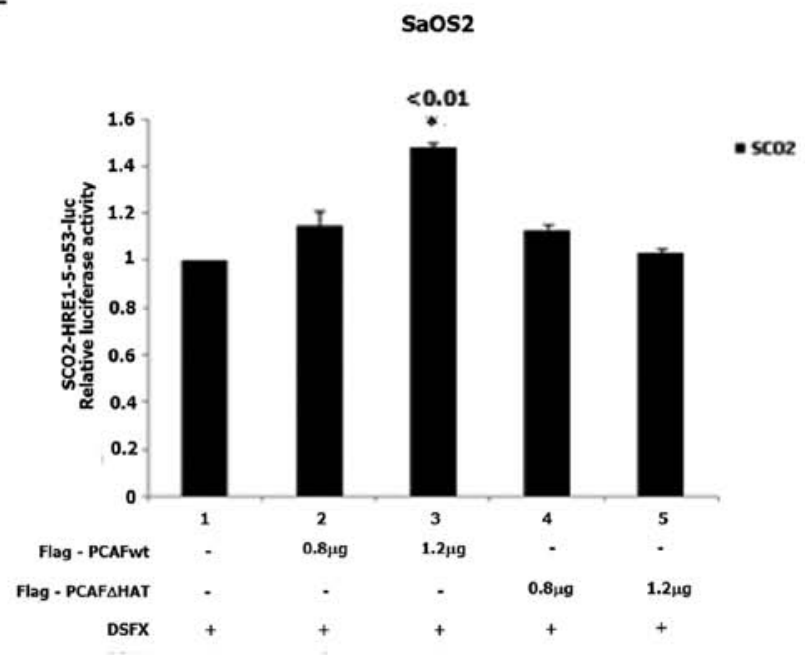

$\mathbf{F}$

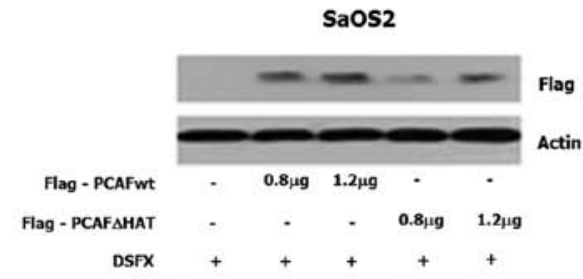

Figure 5. PCAF regulates SCO2 gene expression. SCO2 protein levels were followed in non-treated or DSFX-treated U2OS (A) and SaOS2 (B) cells transfected with either Flag-PCAFwt or Flag-PCAFAHAT. The exogenously expressed proteins were detected using a specific antibody against Flag. Actin was used as a loading control. DSFX-treated U2OS (C) and SaOS2 (D) cells were transfected with increasing amounts of Flag-PCAFwt and Flag-PCAF $\Delta H A T$ along with SCO2-HRE1-5-p53-Luc reporter and subjected to luciferase reporter assays. The protein levels of the transfected Flag-PCAFwt and Flag-PCAF $\Delta$ HAT in U2OS (E) and SaOS2 (F) cells are shown below the reporter assay diagrams. One representative out of three independent experiments is shown in (A), (B), (E) and $(\mathrm{F})$ and the average of three experiments performed in duplicates in (C) and (D).

3 to 1). Increasing amounts of Flag-PCAFwt transfected in $\mathrm{SaOS} 2$ cells resulted in 1.5-fold increase in SCO2-HRE1-5p53bs-Luc activity compared to that exhibited in the SaOS2 cells transfected with the empty vector (Fig. 5E, compare bars 1 to 3). No difference in the SCO2-HRE1-5-p53bs-Luc reporter activity was observed in U2OS and $\mathrm{SaOS} 2$ cells treated with DSFX and ectopically expressing increasing amounts of Flag-PCAFAHAT (Fig. 5B and E, bars 4 and 5). Increasing expression of Flag-PCAFwt and Flag-PCAF $\Delta$ HAT transfected in U2OS and SaOS2 cells is shown in Fig. 5C and $\mathrm{F}$, respectively.

To test whether PCAF plays a role in the regulation of TIGAR gene expression, we followed TIGAR mRNA levels in DSFX-treated U2OS and SaOS2 cells transfected with Flag-PCAFwt or Flag-PCAF $\triangle$ HAT expression vectors. A statistically significant reduction of TIGAR mRNA levels was observed in U2OS cells ectopically expressing Flag-PCAFwt (Fig. 6A, compare bars 2 to 1), whereas TIGAR mRNA levels 
A

U20s
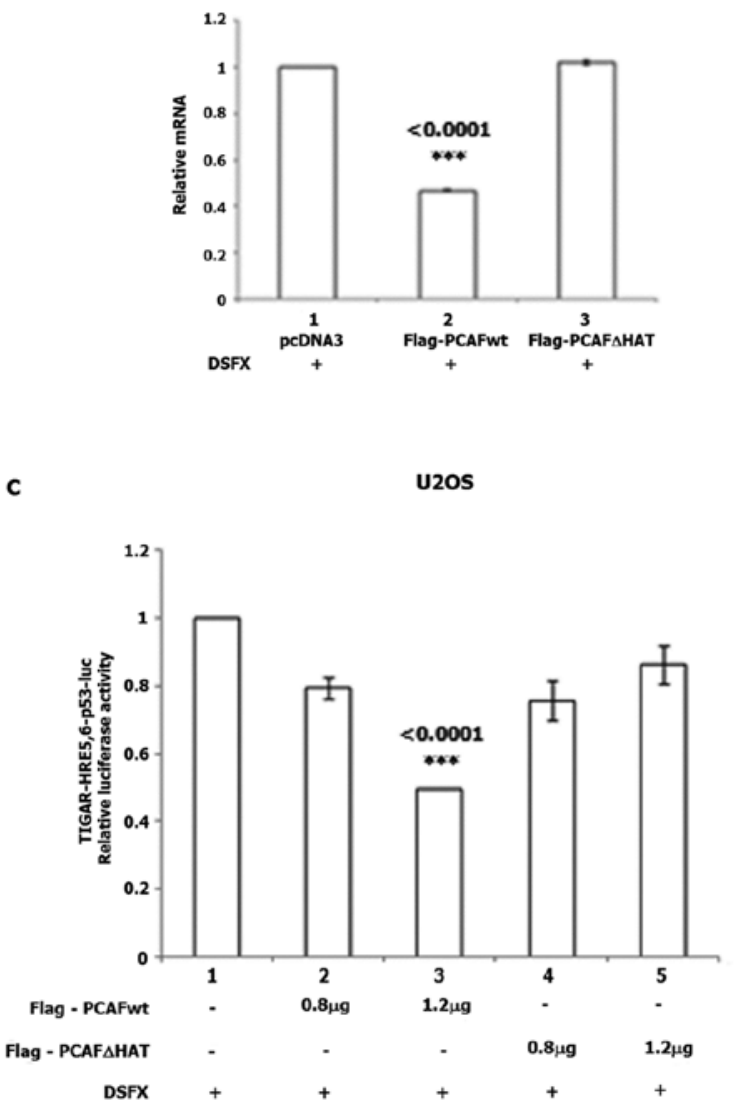

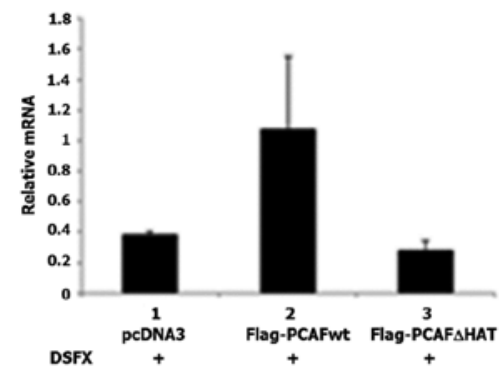

D

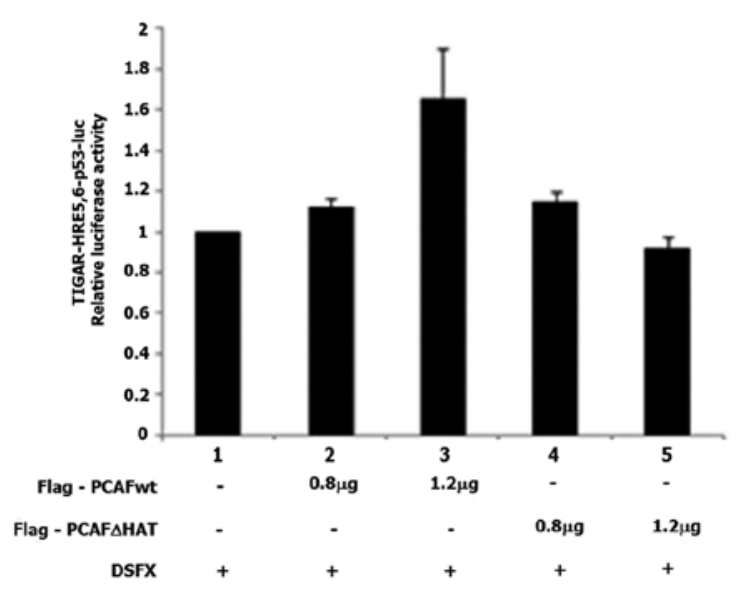

Figure 6. PCAF regulates TIGAR gene expression. DSFX-treated U2OS (A) and SaOS2 (B) cells were transfected with empty vector PCDNA3 or Flag-PCAFwt or Flag-PCAFAHAT and the TIGAR mRNA levels were followed with qRT-PCR. DSFX-treated U2OS (C) and SaOS2 (D) cells were transfected with increasing amounts of Flag-PCAFwt and Flag-PCAFAHAT along with TIGAR-HRE5,6-p53-Luc reporter and subjected to luciferase reporter assay. The average of three independent experiments performed in duplicates are shown in (A-D).

were not affected in Flag-PCAF $\Delta$ HAT-transfected cells (Fig. 6A compare bars 3 to 1). Upregulated TIGAR mRNA levels were detected in SaOS2 cells expressing Flag-PCAFwt (Fig. 6B, compare bars 2 to 1 ) and no effect of Flag-PCAFAHAT (Fig. 6B, compare bars 3 to 1).

Flag-PCAFwt overexpression in DSFX-treated U2OS cells repressed the luciferase activity of the TIGAR-HRE5,6p53bs-Luc reporter (Fig. 6C, compare bars 3 to 1). In DSFX-treated SaOS2 cells overexpression of Flag-PCAFwt induced the luciferase activity of the TIGAR-HRE5,6-p53bsLuc reporter (Fig. 6D, compare bars 3 to 1). Flag-PCAFAHAT overexpression did not affect the luciferase activity of the TIGAR-HRE5,6-p53bs-Luc reporter in either U2OS or SaOS2 cells (Fig. 6C and D respectively, bars 4 and 5). The increasing amounts of Flag-PCAFwt and Flag-PCAF $\Delta$ HAT transfected in U2OS and SaOS2 cells are shown in Fig. 5C and F, respectively. Taken together the results shown in Figs. 5 and 6 support the notion that the HAT activity of PCAF regulates SCO2 and TIGAR cellular levels by fine tuning p53 and HIF- $1 \alpha$ transcriptional activities and the interplay between these transcription factors $(12,16,17)$.

To investigate whether PCAF played a role in the regulation of the expression of the HIF-1 $\alpha$ transcriptional target gene $P F K F B 3$ the protein levels of this glycolytic regulator were followed in U2OS and SaOS2 cells overexpressing either Flag-
PCAFwt or Flag-PCAFAHAT in the presence or absence of DSFX. Reduced PFKFB3 protein levels were observed in the Flag-PCAFAHAT compared to Flag-PCAFwt overexpressing and DSFX-treated U2OS cells (Fig. 7A, compare lanes 4 to 2 and Fig. 7C, compare black bars 2 to 1). In SaOS2 cells the HAT activity of PCAF exhibited the opposite effect to that observed in U2OS on the PFKFB3 protein levels (Fig. 7B, compare lanes 4 to 2 and Fig. 7D, compare black bars 2 to 1 ).

Functional significance of the HAT activity of PCAF in the regulation of cellular energy metabolism. The functional significance of the regulation of SCO2 and TIGAR gene expression mediated by the acetyltransferase activity of PCAF was investigated by assessing the lactate levels produced by cells transfected with either Flag-PCAFwt or Flag-PCAFDHAT. The lactate efflux observed in DSFX-treated cells was normalised to that produced by non-treated cells. Increased lactate production by DSFX-treated U2OS cells overexpressing Flag-PCAFwt was observed compared to that estimated in these cells expressing Flag-PCAFAHAT under the same conditions (Fig. 8A, compare white bars 2 with 3 ). The opposite was the case in SaOS2 cells where overexpression of Flag-PCAFwt led to reduced lactate efflux in DSFX-treated versus non-treated cells whereas transfection of Flag-PCAF $\Delta$ HAT resulted in increased production of lactate in hypoxia mimicking conditions (Fig. 8A, compare 
A

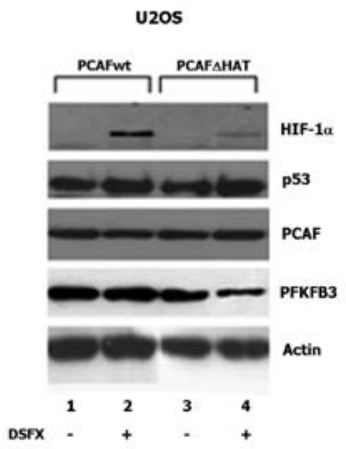

C

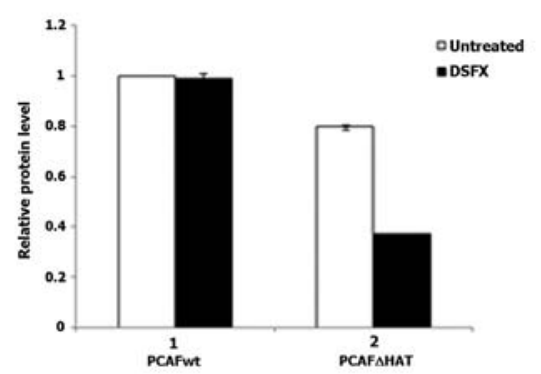

B

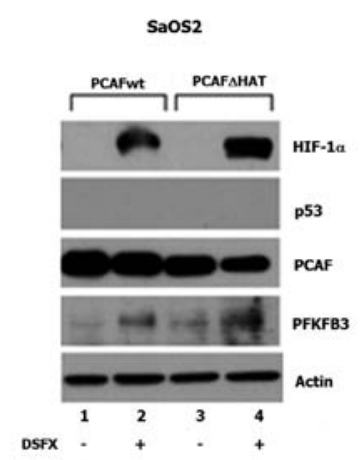

D

Figure 7. PCAF regulates PFKFB3 protein levels. U2OS (A) and SaOS2 (B) cells transfected with Flag-PCAFwt or Flag-PCAF $\triangle H A T$ were left untreated or treated with DSFX and subjected to western blot analysis. Densitometric analysis of PFKFB3 protein levels in U2OS is shown in (C) and in SaOS2 cells in (D).

A

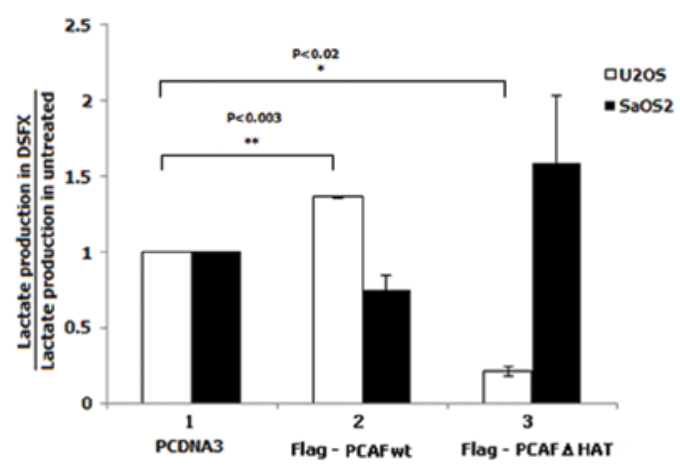

C

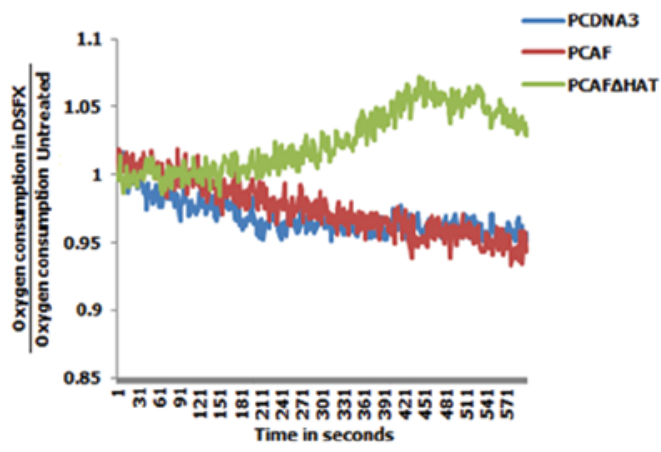

B

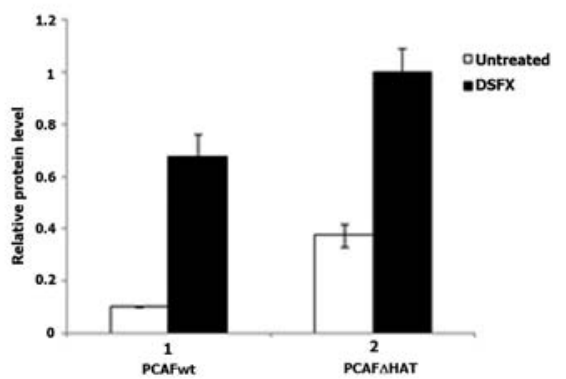

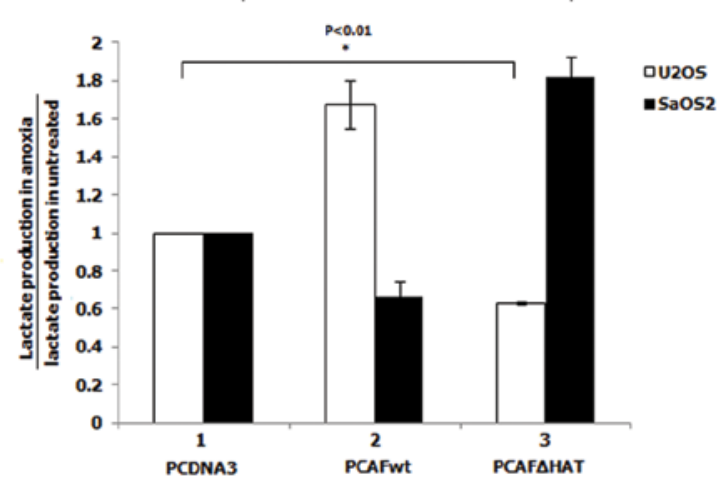

D

SaOS2

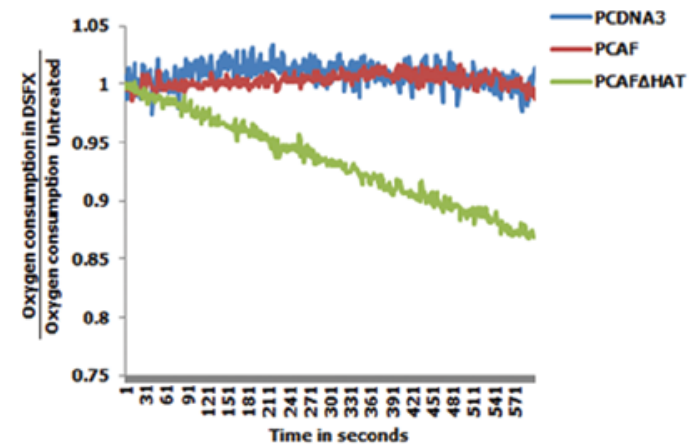

Figure 8. Lactate production and oxygen consumption are differentially regulated by the HAT activity of PCAF in U2OS and SaOS2 cells. Lactate production was assessed in DSFX-treated cells (A) or cells incubated in the anoxic chamber (B). U2OS (white bars) and SaOS2 (black bars) cells were transfected with either PCDNA3 (1) or PCAFwt (2) or PCAF- $\triangle \mathrm{HAT}$ (3) and lactate production was measured as described in Materials and methods. Oxygen consumption was performed in U2OS (C) and SaOS2 (D) cells transfected with PCDNA3 (blue), or PCAFwt (red) or PCAF $\triangle$ HAT (green). Asterisks indicate statistical significance of the results. 


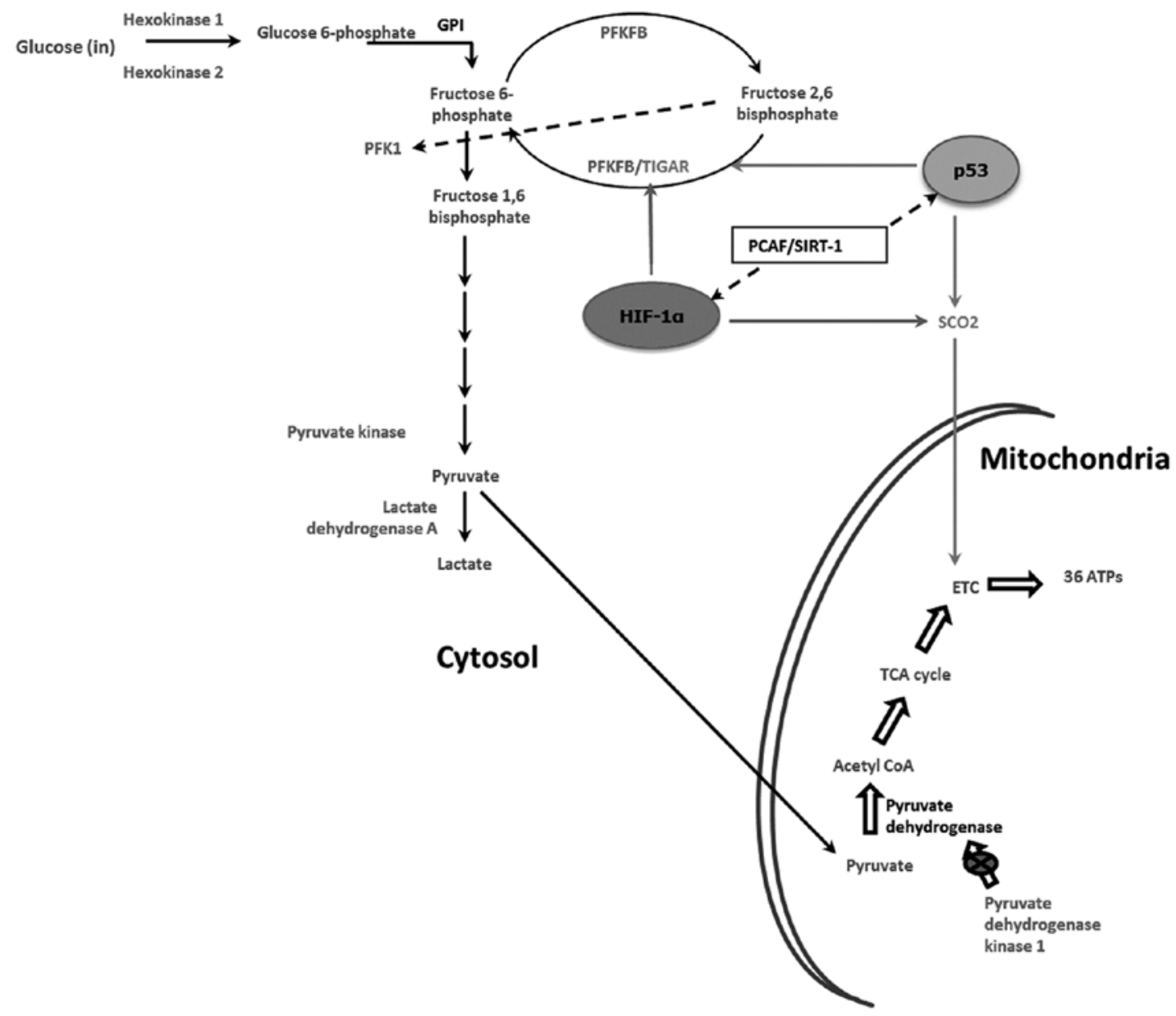

Figure 9. Schematic diagram illustrating the potential regulation of cellular energy metabolism mediated by PCAF. The balance between PCAF and Sirt-1 activity fine tunes the HIF-1 and p53 transcriptional activity and target selectivity regulating TIGAR and SCO2 gene expression thereby orchestrating glycolysis and OXPHOS pathways of cellular energy production under diverse environmental conditions.

black bars 2 with 3). Lactate production measured in U2OS and $\mathrm{SaOS} 2$ cells incubated in an anoxic chamber presented similar results with those obtained from DSFX-treated cells (compare Fig. 8A with B). These findings indicated that the HAT activity of PCAF increased glycolysis in hypoxic $\mathrm{U} 2 \mathrm{OS}$ cells and reduced it in $\mathrm{SaOS} 2$ cells corresponding with downregulated TIGAR gene expression in DSFX-treated and Flag-PCAFwt expressing U2OS cells and upregulated TIGAR gene expression in SaOS2 cells under these conditions (Fig. 6). Thus the differential lactate production in U2OS and SaOS2 cells could be mechanistically explained at least in part by the opposite effect of PCAF on the TIGAR gene expression in the two cell lines (Fig. 6).

Inability to produce energy through OXPHOS in cancer cells is compensated by increased glycolysis (26). Oxygen uptake is an indicator of the ability of the cells to produce energy through the OXPHOS pathway (11). The role of the HAT activity of PCAF in the regulation of OXPHOS was studied by quantifying the ability of Flag-PCAFwt, Flag-PCAFAHAT or PCDNA3 empty vector transfected in U2OS or SaOS2 cells to uptake oxygen (Fig. 8C and D, red, green and blue lines respectively). The oxygen consumption in DSFX-treated and non-treated cells was calculated as described in Materials and methods. While the lactate production of DSFX-treated U2OS cells transfected with Flag-PCAFAHAT was decreased compared to these cells transfected with Flag-PCAFwt under the same conditions (Fig. 8A, compare white bars 3 with 2), the oxygen consumption was found to be increased in the same conditions (Fig. 8C, compare green line with red and blue lines). In contrast to U2OS cells, DSFX-treated SaOS2 cells overexpressing Flag-PCAF $\Delta$ HAT consumed less oxygen compared to cells transfected with Flag-PCAFwt (Fig. 8D, compare green line with red and blue lines). Taken together results shown in Fig. 8 indicate that the HAT activity of PCAF plays an important role in determining the pathway of cellular energy production in hypoxia mimicking conditions.

\section{Discussion}

Proliferation status and physiological conditions are some of the factors determining the cellular energy demands. Among the transcription factors that orchestrate the pathways of cellular energy metabolism to harmonize cell cycle progression and energy metabolism with environmental physiological conditions is the tumour suppressor p53 (27). Inactivation of p53 results in substantial OXPHOS deficiency (11) and increased dependence on glycolysis accompanied by elevated lactate production (28). The p53 mediated switch from OXPHOS to glycolysis is in part implemented by the transcriptional regulation of the expression of a number of genes involved in both 
OXPHOS and glycolysis by this transcription factor (11). For example, p53 acting in a cell type-dependent mode stimulates the expression of genes essential for the completion of the OXPHOS pathway such as SCO2 (11) and inhibits glycolysis by repressing the glucose transporters GLUT-1 and GLUT-4 (29).

In cancer tissues the deprivation of cellular oxygen within hypoxic regions contributes to the shift to glycolysis and eventually tumour cell growth. In response to low oxygen concentration the gene expression of a variety of metabolic enzymes such as GLUT and the regulatory bifunctional PFKFB3 enzyme is directed by the HIF family of transcription factors $(30,31)$. Dramatic increase of the ratio of kinase:phosphatase activity of the HIF-1 $\alpha$ transcription target PFKFB3 in hypoxic conditions results in increased F2,6BP concentration in hypoxic solid tumours $(31,32)$. The homologue to the bisphosphatase domain of the PFK2/FBPase2 gene TIGAR has been identified as a p53 transcriptional target (16) suggesting that the reduction of the phosphatase activity of FBPase2 in hypoxia and increased glycolysis could be exaggerated as a result of active repression of TIGAR gene expression under these conditions. In addition, HIF- $1 \alpha$ mediated downregulation of mitochondrial oxygen consumption and preservation of the Crabtree effect might be the result of differential modulation of $\mathrm{SCO} 2$ gene expression in hypoxia (6).

In order to identify p53 and HIF-1 $\alpha$ common transcription target genes involved in energy production pathways we tested the possibility the gene expression of the known p53 targets $S C O 2$ and TIGAR was under HIF-1 $\alpha$ control. We followed the mRNA and protein levels of these genes in hypoxia mimicking conditions, which revealed that both $S C O 2$ and TIGAR cellular levels were differentially regulated in normoxia compared to hypoxia mimicking conditions (Fig. 1). To find out whether HIF-1 $\alpha$ was one of the factors involved in the regulation of $S C O 2$ and TIGAR mRNA and protein levels we searched for the existence of potential HRE sites within the regulatory region of the promoters of both these genes (Fig. 2) and tested the activity of these sites employing luciferase reporter and chromatin immunoprecipitation assays, which supported the notion that $\mathrm{SCO} 2$ and TIGAR were under HIF-1 transcriptional control (Figs. 3 and 4).

PCAF has been shown to acetylate both HIF- $1 \alpha$ and p53 and coordinate their selective recruitment to pro-survival or apoptotic genes such as p21 and BID thereby regulating cell cycle arrest and apoptosis in hypoxic conditions (17). Given the fact that cell cycle regulation and metabolism share common regulatory pathways (23) and PCAF is a co-factor regulating cell growth effects mediated by both p53 and HIF-1 $\alpha$ (17), we expanded our studies to investigate whether PCAF was involved in the fine tuning of p53 and HIF- $1 \alpha$ mediated effects on metabolism. Luciferase reporter assays pointed out that the HAT activity of PCAF was involved in the HIF- $1 \alpha$ mediated regulation of the expression of SCO2 (Fig. 5) and TIGAR (Fig. 6) gene expression indicating a role of PCAF mediated acetylation as a major regulator for the determination of the pathway of cellular energy production in hypoxia mimicking conditions. Furthermore, downregulation of PFKFB3 in U2OS cells overexpressing Flag-PCAF $\triangle H A T$ in hypoxia mimicking conditions and upregulation of this enzyme in $\mathrm{SaOS} 2$ under the same conditions (Fig. 7) implied that the HAT activity of
PCAF regulated both TIGAR (Fig. 6) and PFKFB3 (Fig. 7) cellular levels. Given the fact that TIGAR shares structural and functional similarity with the phosphatase domain of PFKFB3, this result suggested that TIGAR might compensate for the lack of phosphatase activity of PFKFB in hypoxia mimicking conditions.

The interplay of the two main bioenergetic pathways OXPHOS and glycolysis mediated by the HAT activity of PCAF was investigated by analysing lactate production and oxygen consumption in cells overexpressing Flag-PCAFwt or Flag-PCAF $\triangle H A T$ variants (Fig. 8). Results shown in Fig. 8A and B suggested that Flag-PCAFwt overexpressing U2OS cells depend more on glycolysis in hypoxia mimicking and anoxic conditions than untransfected or Flag-PCAF $\Delta$ HAT expressing cells under the same conditions (Fig. 8A and B). In contrast, in hypoxia mimicking or anoxic conditions $\mathrm{SaOS} 2$ cells overexpressing Flag-PCAFwt exhibited higher lactate level production than those transfected with Flag-PCAF $\triangle H A T$ (Fig. 8A and B). Increased ability of Flag-PCAF $\triangle$ HAT overexpressing U2OS cells to uptake oxygen in hypoxia mimicking conditions (Fig. 8C) could be indicative of elevated capacity of these cells to produce energy through OXPHOS. In addition, the increased lactate production observed in U2OS cells overexpressing Flag-PCAF $\triangle H A T$ comply with the reduced oxygen consumption observed in these cells (Fig. 8A, B and D). Reduced oxygen uptake could be an indication of reduced oxidative phosphorylation, but the increased oxygen consumption may or may not indicate increased OXPHOS (33).

Collectively, the results presented provide evidence to support a role for the HAT activity of PCAF, at least in part, in determining the pathway of cellular energy production in $\mathrm{U} 2 \mathrm{OS}$ and $\mathrm{SaOS} 2$ cells by fine tuning the crosstalk between p53 and HIF-1 $\alpha$ (Fig. 9).

\section{Acknowledgements}

We would like to thank Professor K. Vousden, Professor P.M. Hwang and Dr I. Talianidis for providing reagents used in this study.

\section{References}

1. Fantin VR, St-Pierre J and Leder P: Attenuation of LDH-A expression uncovers a link between glycolysis, mitochondrial physiology, and tumor maintenance. Cancer Cell 9: 425-434, 2006.

2. Vander Heiden MG, Plas DR, Rathmell JC, Fox CJ, Harris MH and Thompson CB: Growth factors can influence cell growth and survival through effects on glucose metabolism. Mol Cell Biol 21: 5899-5912, 2001.

3. Olovnikov IA, Kravchenko JE and Chumakov PM: Homeostatic functions of the p53 tumor suppressor: Regulation of energy metabolism and antioxidant defense. Semin Cancer Biol 19: 32-41, 2009.

4. Sonveaux P, Végran F, Schroeder T, et al: Targeting lactatefueled respiration selectively kills hypoxic tumor cells in mice. J Clin Invest 118: 3930-3942, 2008.

5. Kroemer $\mathrm{G}$ and Pouyssegur J: Tumor cell metabolism: cancer's Achilles' heel. Cancer Cell 13: 472-482, 2008.

6. Papandreou I, Cairns RA, Fontana L, Lim AL and Denko NC: HIF-1 mediates adaptation to hypoxia by actively downregulating mitochondrial oxygen consumption. Cell Metab 3: 187-197, 2006.

7. Semenza G: HIF-1 mediates the Warburg effect in clear cell renal carcinoma. J Bioenerg Biomembr 39: 231-234, 2007.

8. Tennant DA, Duran RV, Boulahbel H and Gottlieb E: Metabolic transformation in cancer. Carcinogenesis 30: 1269-1280, 2009. 
9. Vousden KH and Ryan KM: p53 and metabolism. Nat Rev Cancer 9: 691-700, 2009.

10. Shen L, Sun X, Fu Z, Yang G, Li J and Yao L: The fundamental role of the $\mathrm{p} 53$ pathway in tumor metabolism and its implication in tumor therapy. Clin Cancer Res 18: 1561-1567, 2012.

11. Matoba S, Kang J-G, Patino WD, et al: p53 regulates mitochondrial respiration. Science 312: 1650-1653, 2006.

12. Bensaad K, Cheung EC and Vousden KH: Modulation of intracellular ROS levels by TIGAR controls autophagy. EMBO J 28 : 3015-3026, 2009.

13. Stiburek L and Zeman J: Assembly factors and ATP-dependent proteases in cytochrome c oxidase biogenesis. Biochim Biophys Acta 1797: 1149-1158, 2010.

14. Jaksch M, Ogilvie I, Yao J, et al: Mutations in SCO2 are associated with a distinct form of hypertrophic cardiomyopathy and cytochrome c oxidase deficiency. Hum Mol Genet 9: 795-801, 2000.

15. Li H and Jogl G: Structural and biochemical studies of TIGAR (Tp53-induced glycolysis and apoptosis regulator). J Biol Chem 284: 1748-1754, 2009.

16. Bensaad K, Tsuruta A, Selak MA, et al: TIGAR, a p53-inducible regulator of glycolysis and apoptosis. Cell 126: 107-120, 2006.

17. Xenaki G, Ontikatze T, Rajendran R, et al: PCAF is an HIF-1[alpha] co-factor that regulates p53 transcriptional activity in hypoxia. Oncogene 27: 5785-5796, 2008.

18. Lim J-H, Lee Y-M, Chun Y-S, Chen J, Kim J-E and Park J-W: Sirtuin 1 modulates cellular responses to hypoxia by deacetylating hypoxia-inducible factor $1 \alpha$. Mol Cell 38: 864-878, 2010

19. Fulco M, Schiltz RL, Iezzi S, et al: Sir2 regulates skeletal muscle differentiation as a potential sensor of the redox state. Mol Cell 12: 51-62, 2003

20. Motta MC, Divecha N, Lemieux M, et al: Mammalian SIRT1 represses forkhead transcription factors. Cell 116: 551-563, 2004.

21. Haigis MC and Sinclair DA: Mammalian sirtuins: biological insights and disease relevance. Annu Rev Pathol 5: 253-295, 2010 .
22. Sohda M, Ishikawa $\mathrm{H}$, Masuda N, et al: Pretreatment evaluation of combined HIF-1 $1 \alpha$, p53 and p 21 expression is a useful and sensitive indicator of response to radiation and chemotherapy in esophageal cancer. Int J Cancer 110: 838-844, 2004.

23. Fritz V and Fajas L: Metabolism and proliferation share common regulatory pathways in cancer cells. Oncogene 29: 4369-4377, 2010.

24. Soutoglou E, Katrakili N and Talianidis I: Acetylation regulates transcription factor activity at multiple levels. Mol Cell 5: 745-751, 2000.

25. Demonacos C, Krstic-Demonacos M and La Thangue NB: A TPR motif co-factor contributes to p300 activity in the p53 response. Mol Cell 8: 71-84, 2001.

26. Wallace DC: Bioenergetics, the origins of complexity, and the ascent of man. Proc Natl Acad Sci USA 107: 8947-8953, 2010.

27. Bensaad K and Vousden KH: p53: new roles in metabolism. Trends Cell Biol 17: 286-291, 2007.

28. Zhou S, Kachhap S and Singh KK: Mitochondrial impairment in p53-deficient human cancer cells. Mutagenesis 18: 287-292, 2003.

29. Schwartzenberg-Bar-Yoseph F, Armoni M and Karnieli E: The tumor suppressor p53 down-regulates glucose transporters GLUT1 and GLUT4 gene expression. Cancer Res 64: 2627-2633, 2004.

30. Formenti F, Constantin-Teodosiu D, Emmanuel Y, et al: Regulation of human metabolism by hypoxia-inducible factor. Proc Natl Acad Sci USA 107: 12722-12727, 2010.

31. Yalcin A, Telang S, Clem B and Chesney J: Regulation of glucose metabolism by 6-phosphofructo-2-kinase/fructose-2,6-bisphosphatases in cancer. Exp Mol Pathol 86: 174-179, 2009.

32. Chesney J: 6-phosphofructo-2-kinase/fructose-2,6-bisphosphatase and tumor cell glycolysis. Curr Opin Clin Nutr Metab Care 9: 535-539, 2006

33. Seyfried T and Shelton L: Cancer as a metabolic disease. Nutr Metab 7: 7, 2010. 